\title{
BMJ Open Bugs and Brains, the Gut and Mental Health Study: a mixed-methods study investigating microbiota composition and function in anxiety, depression and irritable bowel syndrome
}

\author{
Carra A Simpson (D) , ${ }^{1,2}$ Orli S Schwartz, ${ }^{3}$ Djamila Eliby, ${ }^{1,2}$ Catherine A Butler, ${ }^{4}$ \\ Katherine Huang, ${ }^{5}$ Neil O'Brien-Simpson, ${ }^{4}$ Bridget L Callaghan, ${ }^{2,6}$ \\ Stuart G Dashper, ${ }^{4}$ Paul R Gooley, ${ }^{5}$ Sarah Whittle, ${ }^{1,2}$ Nick Haslam, ${ }^{1}$ \\ Julian G Simmons ${ }^{1,2}$
}

To cite: Simpson CA, Schwartz OS, Eliby D, et al. Bugs and Brains, the Gut and Mental Health Study: a mixed-methods study investigating microbiota composition and function in anxiety, depression and irritable bowel syndrome. BMJ Open 2021;11:e043221. doi:10.1136/ bmjopen-2020-043221

- Prepublication history and additional material for this paper is available online. To view these files, please visit the journal online (http://dx.doi.org/10. 1136/bmjopen-2020-043221).

Received 28 July 2020 Revised 23 February 2021 Accepted 23 February 2021

Check for updates

(C) Author(s) (or their employer(s)) 2021. Re-use permitted under CC BY-NC. No commercial re-use. See rights and permissions. Published by BMJ.

For numbered affiliations see end of article.

Correspondence to

Carra A Simpson;

carra.simpson@unimelb.edu.au

\section{ABSTRACT}

Introduction Research has highlighted relationships between the micro-organisms that inhabit our gastrointestinal tract (oral and gut microbiota) with host mood and gastrointestinal functioning. Mental health disorders and functional gastrointestinal disorders co-occur at high rates, although the mechanisms underlying these associations remain unclear. The Bugs and Brains Study aims to investigate complex relationships between anxiety/ depression and irritable bowel syndrome (IBS) in two ways. First, its primary component will compare the gut and oral microbiota in females with anxiety/depression and/or IBS relative to controls, and investigate underlying physiological, endocrine and immune factors, as well as associations with diet and psychosocial factors. In an ancillary component, the study will also investigate gastrointestinal and mental health symptoms in a larger sample, and explore relationships with diet, exercise, oral health, substance use, medical history, early life adversity and psychosocial factors.

Methods and analysis The Bugs and Brains Study aims to recruit 160 females to the primary component: (1) 40 controls; (2) 40 participants with a depressive/anxiety disorder, but no IBS; (3) 40 participants with IBS, but no depressive/anxiety disorder and (4) 40 participants with both depressive/anxiety disorder and IBS. Participation is completed within 1 month, and involves comprehensive questionnaires, anthropometrics, a diagnostic clinical interview, collection of two saliva samples, and stool, urine and hair samples. This study aims to use a systems biology approach to characterise oral and gut microbial composition and function using 16S rRNA gene sequencing and nuclear MR spectroscopy. As part of the ancillary component, it will collect questionnaire data from 1000 participants aged 18-40 years, capturing mental health, gastrointestinal health, oral health, diet and psychosocial factors.

Ethics and dissemination Approval was granted by the University of Melbourne Human Research Ethics Committee (\#1749221). All participants voluntarily provided informed consent. Results will be published in peer-reviewed journals and presented at scientific conferences.
Strengths and limitations of this study

- The Bugs and Brains Study will compare oral and gut microbiota composition and function using nextgeneration sequencing and nuclear MR spectroscopy in individuals with anxiety/depression, irritable bowel syndrome (IBS), both anxiety/depression and IBS, and controls, as defined by clinical diagnosis and strict inclusion criteria.

- It will assess diet, medical history, gastrointestinal health, early life adversity, mental and physical health symptoms using detailed questionnaires and clinical interview, and investigate associations between symptoms, microbiota, metabolome, and the immune and endocrine systems.

- It will also investigate gastrointestinal and mental health symptoms in a large sample, and examine relationships with diet, exercise, oral health, substance use, medical history, early life adversity and psychosocial factors.

- Due to the possible effects of host sex, age and substance use on microbial outcomes, this study chose to recruit females aged 18-40 who were nonsmokers, medication free and without a substance abuse disorder; the generalisability of this study is therefore limited to this specific population.

- This study is cross-sectional in nature and employs 16S rRNA gene sequencing; future research should investigate these associations using deeper resolution sequencing (ie, shotgun metagenomics), examine relationships longitudinally to disentangle causes and effects, and extend investigations to male participants.

\section{INTRODUCTION}

Micro-organisms have co-existed alongside humans throughout our evolution, with between 20 and 30 trillion microbial cells occupying ecological niches within and 
on the human body. It is therefore unsurprising that bacteria residing throughout the gastrointestinal (GI) tract are implicated in host endocrine, immune, and nervous system functioning. ${ }^{1}$ Microbes produce neuroactive compounds, including catecholamines (eg, norepinephrine, dopamine), GABA, histamine, serotonin, and acetylcholine, each of which plays a critical role in human physiological functioning. ${ }^{12}$ Multiple GI bacteria and their metabolic products also facilitate peristaltic waves to aid with digestion, ${ }^{3}$ contribute to fermentation of dietary carbohydrates, maintain host membrane function, and produce essential amino acids and vitamins. ${ }^{2}{ }^{4}$ Resident bacterial species also prime the immune system in the early stages of life, teaching our bodies what is 'foreign' rather than commensal. ${ }^{2}$ The gut microbiota (ie, the collection of microorganisms in the GI tract) is therefore essential to normal development.

As we come to appreciate the essential role microorganisms play in host physiology, so too have we opened a novel direction for investigation in conditions with no current clear aetiology. Among these are irritable bowel syndrome (IBS), depression, and anxiety disorders. IBS is the most common functional GI disorder, affecting between $7 \%$ and $21 \%$ of individuals worldwide. ${ }^{5}$ Similarly, depression remains the highest contributor to non-fatal health burden, and approximately 264 million individuals live with an anxiety disorder globally. ${ }^{6}$ These three conditions frequently co-occur ${ }^{7}$ : approximately $84 \%$ of patients with IBS also have a depressive disorder and $44 \%$ an anxiety disorder. ${ }^{89}$ Moreover, $45 \%$ of patients with anxiety and $30 \%$ of patients with a depressive disorder develop IBS. ${ }^{71011}$ The mechanisms underlying this comorbidity remain unclear.

The complex interplay between host physiology and microbiota promises novel insight into these conditions. Research has revealed that anxiety, depression, and IBS share several gut microbial alterations, including decreased overall microbial diversity and a higher relative abundance of proinflammatory and toxigenic species. ${ }^{12-15}$ Moreover, research indicates alterations may exist along a continuum, in that participants with comorbid depression and IBS have gut microbiota alterations simply more pronounced than those presenting with one disorder, including higher proteobacteria, Prevotella/Prevotellaceae, Bacteroides and lower Lachnospiraceae. ${ }^{10}{ }^{16-18}$ These implicated taxa further build on the evidence of altered microbial and host immune system interactions, as the taxa reported to have a higher relative abundance in anxiety, depression, and IBS are associated with inflammation, whereas a loss of species that secrete anti-inflammatory metabolic products has also been observed. ${ }^{14}$ No studies have compared the gut microbiota of participants with anxiety relative to IBS, although studies have identified correlations between bacterial taxa and anxiety symptoms among IBS patients. ${ }^{18-20}$

Although research in this area has primarily investigated the composition of the gut microbiota, the entire length of the GI tract harbours resident microorganisms.
At its entryway, the microbiota of the oral cavity is the second most diverse community in the human body, ${ }^{21}$ and research indicates that microbial representation in the mouth is predictive of composition in the lower GI tract. ${ }^{22}$ Oral bacteria can also translocate throughout the body, with significant effects on GI and systemic health. ${ }^{23}$ No known studies have examined the oral microbiota in clinical anxiety, depression or IBS; however, our existing research observed associations between oral microbiota composition with anxiety and depression symptoms. ${ }^{24}$ Characterisation of the oral microbiota in clinical mental health and IBS cohorts therefore warrants investigation.

Several confounding factors have been neglected in the small literature investigating the microbiota in IBS, anxiety, and depression. Due to their effects on microbial composition, it seems reasonable to consider psychotropic medication use, ${ }^{25}{ }^{26}$ smoking, ${ }^{27}$ alcohol consumption, ${ }^{27}$ IBS subtype and severity, ${ }^{15} 18$ as well as biological sex. ${ }^{28}$ Diet, and its interaction with psychosocial factors, is another source of gut microbiota variation that has been insufficiently considered in existing research.$^{29}{ }^{30}$ Finally, the extant literature has not often utilised gold-standard diagnostic measures to examine mental health (ie, clinical interviews), and studies have examined microbiota composition but neglected microbial function. ${ }^{31}$

\section{Aims and objectives}

Bugs and Brains, the Gut and Mental Health Study (the Bugs and Brains Study) aims to characterise the microbial and physiological profiles in anxiety, depression, and IBS; to examine associations between symptoms and confounding variables; and, to investigate varied contributors to disorder presentation (both across and within disorders). In this primary case-control component, key questions relate to the similarities and differences across bacterial, metabolic, endocrine, and immune indices in a well-controlled cohort. Given the associations between biological sex and the microbiota, this study chose to recruit females only and exclude current smokers or participants with a substance abuse disorder. Possible sources of interstudy variation in previous investigations will be considered (ie, symptom severity, medication use, diet). In a large ancillary questionnaire-only component, this research will also investigate how GI and mental health symptoms are associated with diet, exercise, oral health, substance use, medical history, early life adversity, and psychosocial factors. Understanding shared and distinct microbiota relationships in IBS, anxiety, and depression, particularly within a well-characterised sample, has implications in resolving the directionality of comorbidity, and in informing future targeted clinical interventions.

\section{METHODS AND ANALYSIS}

Participants

Primary case-control component

Females aged 18-40 will be assessed for participation in all phases of the Bugs and Brains Study. Recruitment for the 
study began in October 2017 and all phases are expected to be completed by December 2021. The primary casecontrol component will include 160 community-based female volunteers aged $18-40$ who meet eligibility criteria for one of four groups:

1 . Forty controls.

2. Forty participants with a depressive/anxiety disorder, but no IBS.

3. Forty participants with IBS, but no depressive/anxiety disorder.

4. Forty participants with depressive/anxiety disorder and IBS

The study chose to recruit only participants of the female sex due to significantly elevated rates of all three conditions relative to biological males. ${ }^{632}$ All participants are being recruited through online and printed advertisements at the University of Melbourne and using paid Facebook ads. All participants provide informed consent and demonstrate adequate comprehension of English. Participants who complete biological samples are monetarily reimbursed for their time, and participants who complete the ancillary questionnaire-only component are reimbursed with a personalised dietary analysis.

\section{Ancillary questionnaire-only component}

To investigate associations in a larger sample, the ancillary component aims to collect questionnaire data from 1000 participants aged 18-40, capturing mental health, diet, exercise, GI health, oral health, substance use, medical history, emotion regulation, stress, early life adversity, and medical history.

\section{Inclusion criteria for the case-control component}

Participants in the primary component are required to live in Greater Melbourne or Geelong (unless willing to travel to the site of sample collection: Melbourne, Australia). Inclusion and exclusion criteria are presented in table 1.
All participants initially complete the phase 1 screening questionnaire to assess their likely eligibility for one of the four groups. If participants meet screening criteria, they are invited to take part in a psychiatric diagnostic interview to confirm eligibility (phase 2). All ineligible participants, including those who prefer not to complete sample collection, are invited to complete the ancillary questionnaire-only component.

\section{Depression and anxiety group}

Participants in this group must meet current diagnostic criteria for at least one of six depressive and/or anxiety disorders at phase 2, as assessed by the Structured Clinical Interview for Diagnostic and Statistical Manual fifth edition (DSM-5) Research Version (SCID-5-RV ${ }^{33}$;). Specific disorders for inclusion are: major depressive disorder, persistent depressive disorder, social anxiety disorder, panic disorder, agoraphobia and generalised anxiety disorder. Participants with a comorbid specific phobia, obsessive-compulsive or related disorder, traumarelated or stressor-related disorder, sleep disorder and/or externalising disorder, as assessed in the SCID-5-RV, are included if they also meet criteria for one of the six target disorders. Given the possibility that participants may experience a major depressive episode of relatively brief duration, participants with major depressive disorder will also be included if a clinical episode is in partial remission (ie, although full diagnostic criteria are not met, symptoms of the immediately preceding episode are present and have not fully resolved at the time of assessment). Participants in this group must have no lifetime history of IBS and must not meet current diagnostic criteria for IBS or another functional GI disorder.

\section{IBS group}

Participants must meet current criteria for IBS, as assessed by the Rome IV during phase 1 screening

Table 1 Inclusion criteria for full participation in the Bugs and Brains Study (ie, primary case-control component)

\begin{tabular}{|c|c|c|c|c|}
\hline Inclusion criteria & Controls & Depression/anxiety & IBS & Depression/anxiety and IBS \\
\hline Female sex & $\checkmark$ & $\checkmark$ & $\checkmark$ & $\checkmark$ \\
\hline Fluent in English & $\checkmark$ & $\checkmark$ & $\checkmark$ & $\checkmark$ \\
\hline Live in Melbourne or Geelong (Australia) & $\checkmark$ & $\checkmark$ & $\checkmark$ & $\checkmark$ \\
\hline $\begin{array}{l}\text { Meet DSM- } 5 \text { criteria for current Depression or } \\
\text { Anxiety disorder* }\end{array}$ & $x$ & $\checkmark$ & $x$ & $\checkmark$ \\
\hline $\begin{array}{l}\text { Obessive compulsive disorder, specific phobia, } \\
\text { trauma-related or stressor-related disorder, sleep } \\
\text { disorder, externalising disorders }\end{array}$ & $x$ & - & $x$ & - \\
\hline Irritable bowel syndrome (Rome IV) & $x$ & $x$ & $\checkmark$ & $\checkmark$ \\
\hline
\end{tabular}

$\checkmark$, inclusion criteria; $\boldsymbol{x}$, exclusion criteria (current or lifetime history [as assessed by SCID-5-RV]); -=neither exclusion nor inclusion.

*Specific disorders for inclusion are: major depressive disorder, persistent depressive disorder, social anxiety disorder, panic disorder, agoraphobia and generalised anxiety disorder as assessed by the SCID-5-RV.

DSM-5, Diagnostic and Statistical Manual fifth edition; IBS, irritable bowel syndrome; SCID-5, Structured Clinical Interview for the DSM-5, research version. 
questionnaires. $^{34} 35$ The Rome IV assesses GI symptom duration and delineates diagnosis by the prominent altered stool type: constipation, diarrhoea, mixed or an unspecified dominance. Participants in this group must not meet lifetime diagnostic criteria for any of the DSM- 5 disorders mentioned above (including specific phobia, etc).

\section{Comorbid IBS and anxiety/depression group}

Consistent with measures outlined above, participants must currently meet criteria for IBS and diagnosis of at least one of the six primary anxiety and/or depressive disorders.

\section{Control group}

Participants in this group must have no lifetime history of IBS, must not meet current diagnostic criteria for IBS or another functional GI disorder, and must not meet lifetime diagnostic criteria for any of the DSM-5 disorders mentioned above (ie, current or past).

\section{Exclusion criteria for the primary case-control component (all groups)}

Physical health

Participants are excluded from all groups if they report or present with current obesity, hypertension, cardiovascular disease, respiratory disease, diabetes mellitus, liver cirrhosis, fatty liver disease, inflammatory bowel disease, coeliac disease, other autoimmune disease, short bowel syndrome or a history of abdominal surgery that involved cutting into the GI tract. Participants who report daily cigarette smoking, a recent colonoscopy (past 12 months), or who are pregnant/lactating are also excluded.

\section{Mental health}

Participants are excluded if they have a lifetime history of clinical psychotic symptoms or disorder, bipolar disorder, somatic symptom or related disorder, or over the past 12 months meet criteria for either a feeding and eating disorder or an alcohol or substance use disorder (at phase 1 questionnaires or phase 2 SCID-5 interview).

\section{Medication}

Participants are excluded if they report use of antidepressants, anxiolytics, other psychotropics, steroids, probiotics, prebiotics or synbiotics in the past 4 weeks or throughout the duration of their participation. They are also excluded if they have a current bacterial, fungal or viral infection, or if they have used non-steroidal anti-inflammatory drugs or antifungal medications in the past 2 weeks or throughout their participation. If participants report short-term medication use or illness, they are invited to participate when they meet eligibility criteria. Specific diets may be used to alleviate GI symptoms (eg, reduced intake of fermentable oligosaccharides, disaccharides, monosaccharides, and polyols (FODMAPs), casein-free), and therefore, participants without IBS are excluded if they are following a restrictive diet (ie, do not meet IBS criteria, potentially due to alleviation of symptoms via diet).

\section{Inclusion criteria for the ancillary questionnaire-only component}

Participants who are ineligible for the primary casecontrol component, or who elect not to collect biological samples, are invited to complete the ancillary questionnaire-only component. No strict eligibility criteria apply, although all participants are required to provide informed consent and demonstrate adequate comprehension of English. All participants in the casecontrol component complete the same questionnaires. The phases of participation in the Bugs and Brains Study are presented in figure 1.

\section{Phases of participation}

Phase 1: screening questionnaire (all participants)

Participants who provide informed consent are sent a phase 1 questionnaire link online using Qualtrics (Provo, UT). This component takes approximately $20 \mathrm{~min}$ and comprises a brief demographic overview, the Psychiatric Diagnostic Screening Questionnaire ${ }^{36}$, and the Rome IV IBS module. ${ }^{34}$ It also captures participants' regular diet category (eg, omnivore, low FODMAP), medication/ supplement use and alcohol/substance use. Eligible participants are invited for phase 2 screening. Excluded participants are invited to complete phase 4 questionnaires as part of the ancillary component (bypassing phases 2 and 3).

Phase 2: assessment of current and past mental health diagnoses (SCID-5; case-control component only)

Participants invited for the primary case-control component are administered the SCID-5-RV ${ }^{33}$ to further assess eligibility. Interviews are conducted in-person at the University of Melbourne or online via video application. Trained interviewers administer a modified version of the SCID-5-RV to assess the inclusion and exclusion psychiatric disorders, described in detail in online supplemental material. Researchers also collect a hospitalisation and mental health treatment history (eg, psychotherapeutic and pharmacological) and assess functioning using the Global Assessment of Functioning and Social and Occupational Functioning Assessment Scale. ${ }^{37}$ All interviewers hold a tertiary degree in psychological sciences or related fields, are trained in diagnostic psychological interviewing, and are required to complete Management of Clinical Aggression training ${ }^{38}$ and Applied Suicide Intervention Skills Training (LivingWorks). Fortnightly clinical meetings are conducted with the research clinical psychologist (OSS) to maintain interviewer consistency and discuss any risks presented by participants. Interrater reliability will be conducted for at least $20 \%$ of interviews from eligible participants to assess diagnostic-level consistency. 


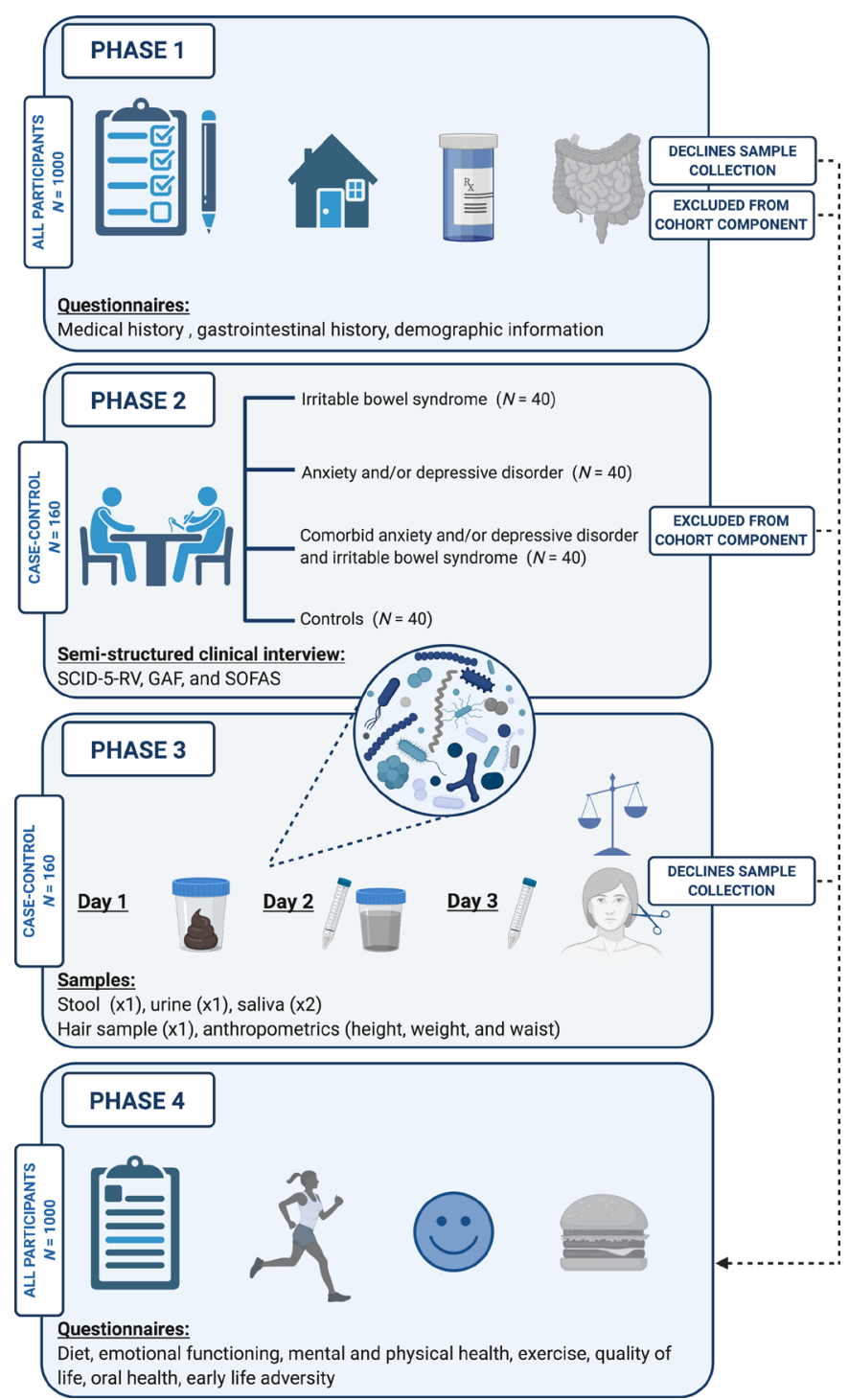

Figure 1 Phases of participation in the Bugs and Brains Study. Participants eligible for the primary case-control component at phases 1 and 2 complete all phases of the study within a month, including biological sample collection ( $n=160 ; n=40$ with IBS, $n=40$ with anxiety and/ or depressive disorder, $\mathrm{n}=40$ with IBS and anxiety and/or depressive disorder, $\mathrm{n}=40$ controls with no lifetime history of either IBS or an anxiety/depressive disorder). Participants who decline sample collection, or are not eligible for the primary component, complete the questionnaire-only ancillary component (phases 1 and 4; $n=1000$ ). GAF, Global Assessment of Functioning; IBS, irritable bowel syndrome; SCID-5-RV, Structured Clinical Interview for the Diagnostic and Statistical Manual (DSM) fifth edition Research version; SOFAS, Social and Occupational Functioning Assessment Scale. Created with BioRender.com.

\section{Phase 3: sample collection and anthropometrics (case-control component only)}

In phase 3 of the study, participants collect biological samples in their own home. Instructions are provided verbally and included in written form with the collection kits. On day 1, a stool sample is collected; on day 2, a urine sample and saliva sample are collected on awakening; on day 3, another saliva sample is collected at awakening. Instructions on sterile storage are provided, and samples are immediately placed in participants' freezers following collection. A home visit is scheduled to pick up the biological samples, collect the hair sample, and take anthropometric measurements (height, weight and waist circumference), ideally within 1 week of collection. Samples are then transported on ice to the university and stored at $-80^{\circ} \mathrm{C}$ until processing.

\section{Faecal sample}

Participants collect a faecal sample at first bowel movement on day 1 of sample collection, but not if they are menstruating or sick. Participants are asked to wait at least 3 days after they cease menses to begin collection, and to not collect samples within 1 week of menstruation, due to the effect of menstrual cycling on endocrine outcomes. ${ }^{39}$ A preliminary literature also describes an oestrogen-gut microbiota axis, ${ }^{40}$ therefore we sought to avoid peaks in oestrogen secretion that may confound microbial analyses. Prior to sample collection, participants are also asked to consume a diet 'normal for them' for at least 2 weeks. Approximately $2.5 \mathrm{~g}$ is collected into sterile faecal collection containers. Participants are instructed not to collect the faecal sample if they have very loose stools, and to wait until they return to a firmer state (ie, Bristol stool chart type 5 or 6 , if diarrhoea is frequent).

\section{Urine sample}

A mid-stream urine sample of approximately $10-20 \mathrm{~mL}$ is collected on day 2 (at first urination) into sterile urine specimen containers (the day after faecal sample collection).

\section{Saliva sample}

Participants collect two $3 \mathrm{~mL}$ saliva samples (by passive drool), one per day across 2 days (day 2 and day 3 ), into $10 \mathrm{~mL}$ Techno Plas sterile centrifuge tubes. Saliva samples are collected at awakening before eating or drinking. Participants are asked to complete a brief saliva record form.

\section{Hair sample}

A hair sample is collected by researchers at participants' homes. Hair sampling is carried out by combing and pinning the surrounding hair with clips, such that a $2 \mathrm{~cm}$ long by $0.5 \mathrm{~cm}$ wide scalp area is isolated on the posterior vertex (back of the head below the inion; $\sim 1 \mathrm{~cm}^{2}$ ). Hair is then bound together (marking the scalp end) and cut with blunt-nose scissors as close to the scalp as possible.

\section{Phase 4: additional questionnaire data (all participants)}

Participants receive the phase 4 questionnaires online via Qualtrics. Phase 4 questionnaires are completed within 24 hours of faecal sample collection. Participants in the ancillary questionnaire-only study complete phase 4 questionnaires following phase 1 (or whichever phase they are excluded from the case-control component). The phase 4 questionnaires cover demographic information (eg, 
country of birth), early life history (eg, mode of birth, breastfeeding), history of gastric infections and scales assessing psychopathology symptoms, stressful life events, early life adversity, diet, emotion regulation, and oral and physical health. The phase 4 questionnaires take approximately $60 \mathrm{~min}$. All questionnaires included in the Bugs and Brains Study are displayed in table 2.

\section{Gut and oral microbiota sequencing}

Amplicon 16S ribosomal ribonucleic acid (rRNA) gene sequencing will be used to estimate participants' microbiota from a faecal sample (gut microbiota) and a saliva sample (oral microbiota). Genomic DNA will be extracted using DNeasy PowerSoil HTP kit (QIAGEN) and amplified using universal prokaryotic $515 \mathrm{~F}$ and $806 \mathrm{R}$ primers targeting the V4 region of the $16 \mathrm{~S}$ gene. ${ }^{41}$ Sequences will be generated using paired-end sequencing on the Illumina MiSeq platform. Should additional funding be secured, shotgun metagenomic sequencing will be performed.

\section{Metabolomics}

Nuclear MR spectroscopy (NMR) will be run on faecal and urine samples to quantify metabolites. If additional funding is obtained, mass spectrometry metabolite assays will also be conducted.

\section{Sample preparation}

\section{Faecal water}

A small proportion of the frozen faecal sample $(0.5-1 \mathrm{~g})$ will be homogenised by vortex in deuterated saline $(0.9 \%$ $\mathrm{NaCl})$ at $1: 5 \mathrm{w} / \mathrm{v}$ before being centrifuged $(3200 \times \mathrm{g})$ at $4^{\circ} \mathrm{C}$ for $15 \mathrm{~min}$. Faecal water will be syringe filtered $(0.22 \mu \mathrm{m}$, $33 \mathrm{~mm}$ diameter) and dispensed into $700 \mu \mathrm{L}$ aliquots.

\section{Urine}

Frozen urine samples will be thawed in ice baths, syringe filtered $(0.22 \mu \mathrm{m}, 33 \mathrm{~mm}$ diameter $)$ and each dispensed into $700 \mu \mathrm{L}$ aliquots.

\section{Metabolite extraction}

Faecal and urine samples will be prepared for NMR analysis using a liquid-liquid extraction technique. ${ }^{42}$ A $250 \mu \mathrm{L}$ aliquot of each faecal water/urine sample will be added to $250 \mu \mathrm{L}$ ice-cold deuterated chloroform and $250 \mu \mathrm{L}$ icecold deuterated methanol and mixed by vortex before being left on ice for $15 \mathrm{~min}$. Samples are then centrifuged $(16000 \times \mathrm{g})$ at $4^{\circ} \mathrm{C}$ for $10 \mathrm{~min}$ to produce a biphasic mixture with a hydrophilic phase of water/deuterated methanol containing polar metabolites and lipophilic phase of deuterated chloroform containing lipophilic compounds.

\section{NMR spectroscopy}

A $297 \mu \mathrm{L}$ sample of the top hydrophilic layer will be added to equal volume of $200 \mathrm{mM}$ sodium phosphate in ${ }^{2} \mathrm{H}_{2} \mathrm{O}(\mathrm{pH} 7.4)$ and $66 \mu \mathrm{L}$ of ${ }^{2} \mathrm{H}_{2} \mathrm{O}$ containing $5 \mathrm{mM} \mathrm{3-(}$ trimethylsilyl)-1-propanesulfonic acid-d6 sodium salt (DSS) and $0.2 \%(\mathrm{w} / \mathrm{v})$ sodium azide to make a total of
$660 \mu \mathrm{L}$. The sample will be mixed by vortex, centrifuged at $16000 \times \mathrm{g}$ for $1 \mathrm{~min}$, and $550 \mu \mathrm{L}$ of supernatant transferred to a 7-inch $5 \mathrm{~mm}$ 507-grade Bruker NMR tube for NMR analysis.

All NMR spectra of faecal water and urine will be collected using Bruker $600 \mathrm{MHz}$ spectrometer set to a constant temperature of $300 \mathrm{~K}$. Standard 1D ${ }^{1} \mathrm{H}$ spectra will be acquired for faecal water using 1D NOESY pulse sequence (recycle delay- $90^{\circ}-\mathrm{t}_{1}-90^{\circ}-\mathrm{t}_{\mathrm{m}}$-acquire) with $\mathrm{t}_{1}$ corresponding to $3 \mu \mathrm{s}, \mathrm{t}_{\mathrm{m}}$ to $10 \mathrm{~ms}$ and the $90^{\circ}$ pulses optimised. Water suppression will be achieved by presaturation of the water signal during $\mathrm{t}_{\mathrm{m}}$ and the recycle delay (4s). Each spectrum will be collected over $64 \mathrm{~K}$ data points and 256 scans with spectral width of $20 \mathrm{ppm}^{43}$

Urine spectra are acquired using the same 1D NOESY pulse sequence where recycle delay correspond to $2 \mathrm{~s}$, $\mathrm{t}_{1}$ to $3 \mu$ s and $\mathrm{t}_{\mathrm{m}}$ to $10 \mathrm{~ms}$. Each spectrum will be collected over $32 \mathrm{~K}$ data points and 64 scans with spectral width of 20 ppm. ${ }^{44}$ Using the Chenomx NMR Suite Professional software package, the free induction decay is multiplied by $0.3 \mathrm{~Hz}$ exponential line broadening and zero-filled before Fourier transformation. Spectra are manually phased, baseline corrected and referenced to DSS with a chemical shift of $0 \mathrm{ppm}$. The compound libraries in the Chenomx software will be used to identify and quantitate metabolites in the NMR $1 \mathrm{D}{ }^{1} \mathrm{H}$ spectra based on their characteristic chemical shifts using $5 \mathrm{mM}$ DSS as an internal chemical shift reference.

\section{Immune and endocrine assays}

Hormones will be assayed from hair and saliva samples to estimate longer and shorter-term endocrine functioning, respectively. The hormones cortisol, dehydroepiandrosterone (DHEA) and testosterone will be assayed to provide an indication of hypothalamic-pituitary-adrenal axis secretion. Systemic inflammation will be estimated using the acute phase $\mathrm{C}$ reactive protein (CRP), as previously described. ${ }^{45}$ Assays for hormones and CRP will be conducted using ELISA kits and performed in duplicate, following manufacturer protocols.

\section{Preprocessing of bacterial sequencing data}

Sequencing reads will be quality filtered, trimmed, denoised and merged using QIIME2. ${ }^{46}$ The DADA2 denoising pipeline will be used for correcting sequence errors and identifying amplicon sequence variants (ASVs, ie, sequences not containing errors). ${ }^{47}$ ASVs will be taxonomically assigned using a Naïve-Bayes classifier against the most recent version of the Human Oral Microbiome Database (saliva) and SILVA databases (faecal). Sequences will be aligned with Multiple Alignment using Fast Fourier Transform (MAFFT) ${ }^{48}$ and phylogeny built using FastTree $2 .{ }^{49}$

\section{Sample size calculation}

A sample of 132 would provide $80 \%$ power at $p=0.01$ to detect an effect size of 0.35 when comparing outcomes across the four groups (ie, anxiety/depression, IBS, 
Table 2 Summary of questionnaire measures included in the Bugs and Brains Study

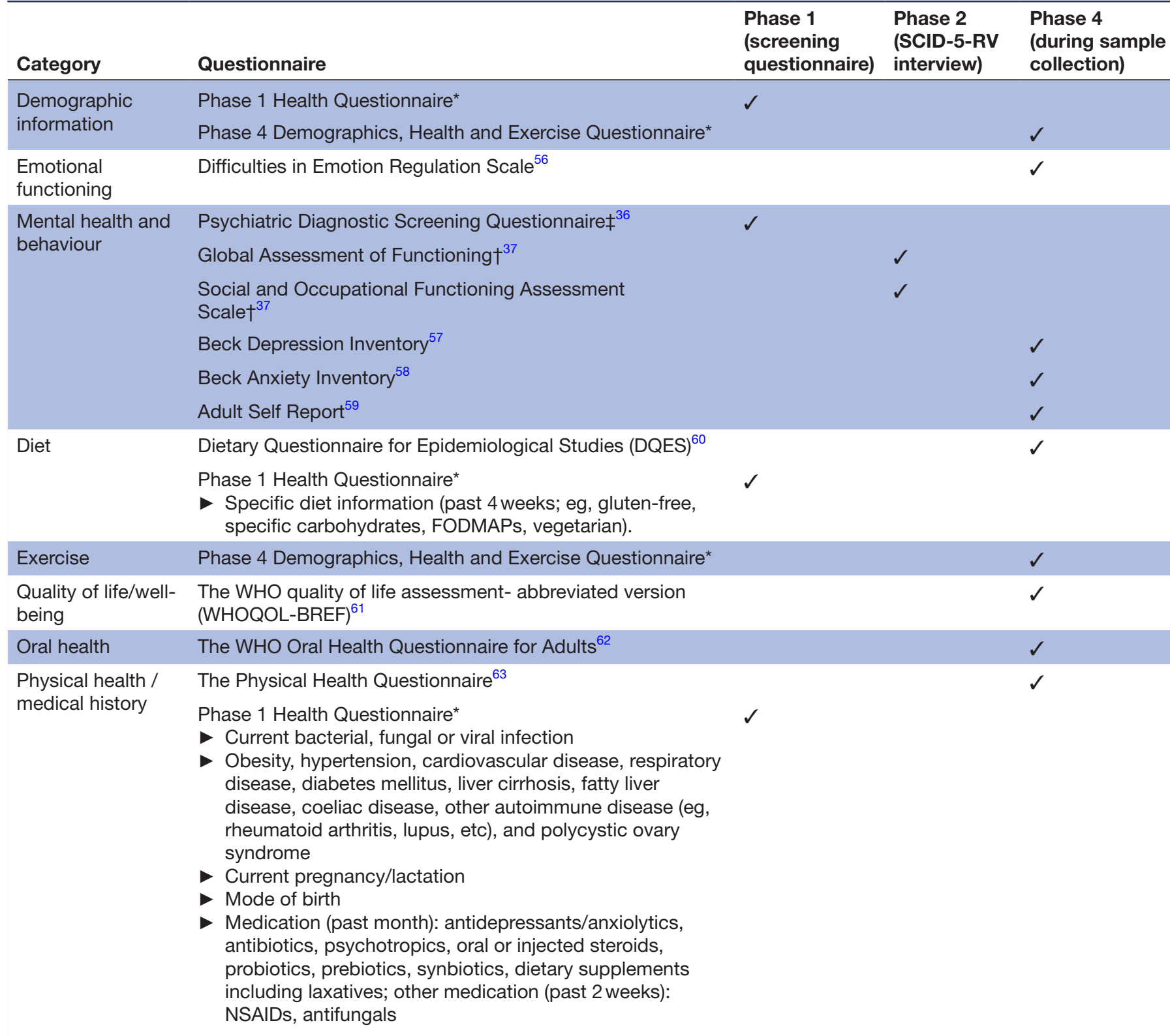

$\begin{array}{ll}\begin{array}{l}\text { Gastrointestinal } \\ \text { health/history }\end{array} & \checkmark \\ & \text { Pome IV Diagnostic Questionnaire } 1 \text { Health Questionnaire* } \\ & \text { Non-surgical procedures related to the digestive system } \\ & \text { (past 6 months): enema, colonoscopy, gastroscopy, } \\ & \text { inflammatory bowel disease (eg, ulcerative colitis, Crohn's } \\ & \text { disease), short bowel syndrome, and lifetime history of } \\ & \text { abdominal surgery (eg, appendectomy, ileostomy, bowel } \\ & \text { surgery, caesarean section, etc) } \\ & \text { Phase 4 Demographics, Health and Exercise Questionnaire* } \\ & \text { Lifetime history of gastric infections: Salmonella, Shigella, } \\ & \text { Campylobacter, Giardiasis, viruses, other }\end{array}$


Table 2 Continued

\begin{tabular}{|c|c|c|c|c|}
\hline Category & Questionnaire & $\begin{array}{l}\text { Phase } 1 \\
\text { (screening } \\
\text { questionnaire) }\end{array}$ & $\begin{array}{l}\text { Phase } 2 \\
\text { (SCID-5-RV } \\
\text { interview) }\end{array}$ & $\begin{array}{l}\text { Phase } 4 \\
\text { (during sample } \\
\text { collection) }\end{array}$ \\
\hline Substance use & $\begin{array}{l}\text { Phase } 1 \text { Health Questionnaire* } \\
\text { Alcohol (past month) } \\
\text { Smoking (past month): tobacco cigarettes, vape, e- } \\
\text { cigarettes containing nicotine } \\
\text { Illicit drug use (past month) }\end{array}$ & $\checkmark$ & & \\
\hline
\end{tabular}

*Designed specifically for the Bugs and Brains Study.

†Measure completed by researchers, rather than participants.

$\ddagger$ Modified version used, see online supplemental material.

DQES, Dietary Questionnaire for Epidemiological Studies; FODMAPs, fermentable oligosaccharides, disaccharides, monosaccharides, and polyols; NSAIDS, non-steroidal anti-inflammatory drugs; SCID-5-RV, Structured Clinical Interview for the Diagnostic and Statistical Manual fifth edition, research version; WHO, World Health Organization.

comorbidity, controls). This estimate is conservative given the effect sizes reported in related research, and our sample size of 160 exceeds this recommendation.

\section{Data analysis}

Proposed analyses will be discipline-specific (microbial, metabolomics, immune and endocrine), but will also use systems biology to integrate the wide range of acquired biological, health and psychosocial data. General linear models will investigate the relationships between mental health, dietary patterns, exercise, GI health, oral health, early life adversity, substance use (including alcohol) and medical history in the questionnaire-only ancillary study. These analyses may inform necessary metadata to consider in analyses of the primary case-control component. Sources of interstudy variation identified in previous investigations of the gut microbiota will be examined. ${ }^{1431}$

A primary aim of the case-control component involves characterising and comparing the microbiota and metabolome in the four participant groups (ie, anxiety/ depression, IBS, comorbidity, controls). Between and within-sample diversity will be conducted using alpha diversity (Shannon and Simpson indices, Faith's phylogenetic diversity, observed number of ASVs (richness)), and beta diversity (PCoA using weighted and unweighted UniFrac distances, examined statistically using PERMANOVA). Differential abundance of microbial taxa will be examined at several levels of the taxonomy (phylum, order, family, genus levels) using packages suited to compositional data: ANCOM-BC ${ }^{50}$ and MaAsLin2, ${ }^{51}$ correcting for multiple comparisons (Benjamini Hochberg False Discovery Rate). Associations between taxa abundance and metadata will also be analysed using these packages. All statistical analyses and graphical representations will be conducted using $\mathrm{R}$, with packages that enable multivariate modelling and visualisations (eg, vegan ${ }^{52}$; phylose $^{53}$; ggplot $2^{54}$; ANCOM-BC ${ }^{50}$; MaAsLin2 ${ }^{51}$ ). It will also integrate the wide range of biological data and adopt a systems biology approach using the package mixOmics. ${ }^{55}$

\section{Ethics and dissemination}

Ethical approval for this project was granted by the University of Melbourne Human Research Ethics Committee (\#1749221). After reading our Plain Language Statement, a comprehensive consent procedure is conducted via telephone with all participants. The voluntary nature of the study is explained, including the limits of confidentiality, the secure and deidentified nature of all data, and the right to withdraw at any time without prejudice or explanation. Verbal consent is recorded at the end of the first phone call, should the participant wish to begin their participation. Written consent is obtained from participants in the case-control component following sample collection.

\section{Patient and public involvement}

There were no funds or time allocated for patient and public involvement, therefore we were unable to engage patients in study design and recruitment. We have invited participants to assist with the development of our dissemination strategy.

\section{Progress to date}

Of the 775 participants who have completed the phase 1 questionnaire since the end of 2017, 211 have met screening criteria and were invited to the SCID-5-RV interview (phase 2). At interview, 53 participants met exclusion criteria relating to psychiatric diagnosis. Several participants were unable to complete kit collection, moved locations, or began exclusionary medications. Accordingly, 140 of 160 participants have completed the case-control component. A total of 352 of the planned 1000 participants have completed the ancillary questionnaire-only component (phases 1 and 4 ).

\section{DISCUSSION}

The high rates of co-occurrence between anxiety, depression and IBS remain poorly understood, although interactions between host physiology and the microbiota provide 
a promising avenue to investigate possible shared underlying mechanisms. The Bugs and Brains Study will be the first research project to investigate and compare the oral and gut microbiota and metabolome of participants with anxiety/depression, IBS, comorbid anxiety/depression and IBS, and controls, as assessed by clinical diagnosis and gold standard criteria. Moreover, it will analyse hypothesised underlying mechanisms, including immune, endocrine and microbial factors, and maintain strict inclusion and exclusion criteria. The questionnaire-only study will examine correlates of GI and mental health symptoms that have been insufficiently examined to date, including diet, exercise, oral health, substance use, medical history, early life adversity and psychosocial factors. Via a comprehensive analysis of symptom-based questionnaire data and detailed characterisation of physiological, microbial and psychological profiles, the Bugs and Brains Study may inform the development of targeted interventions for the millions of individuals living with anxiety, depression and/or IBS.

\section{Author affiliations}

${ }^{1}$ Melbourne School of Psychological Sciences, The University of Melbourne Faculty of Medicine Dentistry and Health Sciences, Melbourne, Victoria, Australia ${ }^{2}$ Melbourne Neuropsychiatry Centre, The University of Melbourne Faculty of Medicine Dentistry and Health Sciences, Melbourne, Victoria, Australia

${ }^{3}$ Centre for Youth Mental Health, Orygen The National Centre of Excellence in Youth Mental Health, Parkville, Victoria, Australia

${ }^{4}$ Centre for Oral Health Research, Melbourne Dental School, Bio21 Institute, The University of Melbourne Faculty of Medicine Dentistry and Health Sciences, Melbourne, Victoria, Australia

${ }^{5}$ Department of Biochemistry and Molecular Biology, The University of Melbourne Bio21 Molecular Science and Biotechnology Institute, Parkville, Victoria, Australia ${ }^{6}$ The Department of Psychology, University of California Los Angeles, Los Angeles, California, USA

\section{Twitter Carra A Simpson @carrasimpson}

Acknowledgements Authors would like to thank all staff, students, and volunteers on the Bugs and Brains Study, as well as all participants who graciously gave their time to this research. Authors report no actual or perceived conflicts of interest.

Contributors JGS conceived the study, with critical input from NH, OSS, SW, CAS, BLC, SGD, PRG, NS-O, CAB, KH and DE. CAS, JGS and KH wrote the manuscript, and CAS devised the analytical strategy. All authors critically revised and approved the final manuscript.

Funding This research is funded by the Melbourne School of Psychological Sciences. Carra Simpson is supported by the Australian Government Research Training Programme. This work was funded by the National Health and Medical Research Council (Career Development Fellowship to SW, ID 1125504).

Competing interests None declared.

Patient consent for publication Not required.

Provenance and peer review Not commissioned; externally peer reviewed.

Supplemental material This content has been supplied by the author(s). It has not been vetted by BMJ Publishing Group Limited (BMJ) and may not have been peer-reviewed. Any opinions or recommendations discussed are solely those of the author(s) and are not endorsed by BMJ. BMJ disclaims all liability and responsibility arising from any reliance placed on the content. Where the content includes any translated material, BMJ does not warrant the accuracy and reliability of the translations (including but not limited to local regulations, clinical guidelines, terminology, drug names and drug dosages), and is not responsible for any error and/or omissions arising from translation and adaptation or otherwise.

Open access This is an open access article distributed in accordance with the Creative Commons Attribution Non Commercial (CC BY-NC 4.0) license, which permits others to distribute, remix, adapt, build upon this work non-commercially, and license their derivative works on different terms, provided the original work is properly cited, appropriate credit is given, any changes made indicated, and the use is non-commercial. See: http://creativecommons.org/licenses/by-nc/4.0/.

ORCID iD

Carra A Simpson http://orcid.org/0000-0002-8281-5881

\section{REFERENCES}

1 Neuman H, Debelius JW, Knight R, et al. Microbial endocrinology: the interplay between the microbiota and the endocrine system. FEMS Microbiol Rev 2015;39:509-21.

2 Kamada N, Seo S-U, Chen GY, et al. Role of the gut microbiota in immunity and inflammatory disease. Nat Rev Immunol 2013;13:321-35.

3 Sikander A, Rana SV, Prasad KK. Role of serotonin in gastrointestinal motility and irritable bowel syndrome. Clin Chim Acta 2009;403:47-55.

4 Martinez FD. The human microbiome. Early life determinant of health outcomes. Ann Am Thorac Soc 2014;11(Suppl 1):S7-12.

5 Lovell RM, Ford AC. Global prevalence of and risk factors for irritable bowel syndrome: a meta-analysis. Clin Gastroenterol Hepatol 2012;10:712-21.

6 World Health Organization. Depression and other common mental health disorders: global health estimates, 2017. Available: http:// apps.who.int/iris/bitstream/handle/10665/254610/WHO-MSDMER-2017.2-eng.pdf;jsessionid=D7B089CDA4A6FD66BD711AE0 E39C4511? sequence=1 [Accessed 17 Jul 2019].

7 Pinto-Sanchez MI, Ford AC, Avila CA, et al. Anxiety and depression increase in a stepwise manner in parallel with multiple FGIDs and symptom severity and frequency. Am J Gastroenterol 2015;110:1038-48.

8 Banerjee A, Sarkhel S, Sarkar R, et al. Anxiety and depression in irritable bowel syndrome. Indian J Psychol Med 2017;39:741-5.

9 Fond G, Loundou A, Hamdani N, et al. Anxiety and depression comorbidities in irritable bowel syndrome (IBS): a systematic review and meta-analysis. Eur Arch Psychiatry Clin Neurosci 2014;264:651-60.

10 Kurokawa S, Kishimoto T, Mizuno S, et al. The effect of fecal microbiota transplantation on psychiatric symptoms among patients with irritable bowel syndrome, functional diarrhea and functional constipation: an open-label observational study. J Affect Disord 2018;235:506-12.

11 Sibelli $A$, Chalder T, Everitt $\mathrm{H}$, et al. A systematic review with metaanalysis of the role of anxiety and depression in irritable bowel syndrome onset. Psychol Med 2016;46:3065-80.

12 Jiang $\mathrm{H}-\mathrm{Y}$, Zhang $\mathrm{X}, \mathrm{Yu} \mathrm{Z}-\mathrm{H}$, et al. Altered gut microbiota profile in patients with generalized anxiety disorder. $J$ Psychiatr Res 2018;104:130-6.

13 Winter G, Hart RA, Charlesworth RPG, et al. Gut microbiome and depression: what we know and what we need to know. Rev Neurosci 2018;29:629-43.

14 Simpson CA, Mu A, Haslam N, et al. Feeling down? A systematic review of the gut microbiota in anxiety/depression and irritable bowel syndrome. J Affect Disord 2020;266:429-46.

15 Pittayanon R, Lau JT, Yuan Y, et al. Gut microbiota in patients with irritable bowel syndrome-a systematic review. Gastroenterology 2019;157:97-108.

16 Aizawa E, Tsuji H, Asahara T, et al. Possible association of Bifidobacterium and Lactobacillus in the gut microbiota of patients with major depressive disorder. J Affect Disord 2016;202:254-7.

17 Liu Y, Zhang L, Wang X, et al. Similar fecal microbiota signatures in patients with diarrhea-predominant irritable bowel syndrome and patients with depression. Clin Gastroenterol Hepatol 2016;14:1602-11.

18 Tap J, Derrien M, Törnblom $\mathrm{H}$, et al. Identification of an intestinal microbiota signature associated with severity of irritable bowel syndrome. Gastroenterology 2017;152:111-23.

19 Labus JS, Hollister EB, Jacobs J, et al. Differences in gut microbial composition correlate with regional brain volumes in irritable bowel syndrome. Microbiome 2017;5:1-17.

20 Sundin J, Rangel I, Fuentes S, et al. Altered faecal and mucosal microbial composition in post-infectious irritable bowel syndrome patients correlates with mucosal lymphocyte phenotypes and psychological distress. Aliment Pharmacol Ther 2015;41:342-51.

21 Deo PN, Deshmukh R. Oral microbiome: unveiling the fundamentals. J Oral Maxillofac Pathol 2019;23:122-8.

22 Turnbaugh PJ, Ley RE, Hamady M, et al. The human microbiome project. Nature 2007;449:804-10. 
23 Olsen I, Yamazaki K. Can oral bacteria affect the microbiome of the gut? J Oral Microbiol 2019;11:1586422.

24 Simpson CA, Adler C, du Plessis MR, et al. Oral microbiome composition, but not diversity, is associated with adolescent anxiety and depression symptoms. Physiol Behav 2020;226:113126.

25 Valles-Colomer M, Falony G, Darzi Y, et al. The neuroactive potential of the human gut microbiota in quality of life and depression. Nat Microbiol 2019;4:623-32.

26 Flowers SA, Ellingrod VL. The microbiome in mental health: potential contribution of gut microbiota in disease and pharmacotherapy management. Pharmacotherapy 2015;35:910-6.

27 Capurso G, Lahner E. The interaction between smoking, alcohol and the gut microbiome. Best Pract Res Clin Gastroenterol 2017;31:579-88.

$28 \mathrm{Ma}$ ZS, Li W. How and why men and women differ in their Microbiomes: medical ecology and network analyses of the Microgenderome. Adv Sci 2019;6:1902054.

29 Dash S, Clarke G, Berk M, et al. The gut microbiome and diet in psychiatry: focus on depression. Curr Opin Psychiatry 2015;28:1-6.

30 Kolodziejczyk AA, Zheng D, Elinav E. Diet-microbiota interactions and personalized nutrition. Nat Rev Microbiol 2019;17:742-53.

31 Simpson CA, Schwartz OS, Simmons JG. The human gut microbiota and depression: widely reviewed, yet poorly understood. J Affect Disord 2020;274:73-5.

32 Canavan C, West J, Card T. The epidemiology of irritable bowel syndrome. Clin Epidemiol 2014;6:71-80.

33 First M, Williams J, Karg R. Structured clinical interview for DSM-5, research version (SCID-5 for DSM-5, research version; SCID-5-RV). Arlington, VA: American Psychiatric Association, 2015.

34 Drossman DA, Hasler WL. Rome IV-Functional Gi disorders: disorders of gut-brain interaction. Gastroenterology 2016;150:1257-61.

35 Drossman DA. Functional gastrointestinal disorders: history, pathophysiology, clinical features and Rome IV. Gastroenterology 2016;150:1262-79.

36 Zimmerman M, Mattia Jl. A self-report scale to help make psychiatric diagnoses: the psychiatric diagnostic screening questionnaire. Arch Gen Psychiatry 2001;58:787-94.

37 American Psychiatric Association. American psychiatric association: diagnostic and statistical manual of mental disorders, fourth edition, text revision (DSM-IV-TR). Washington, DC: American Psychiatric Association, 2000.

38 Gerdtz MF, Daniel C, Dearie V. The Management of Clinical Aggression-Rapid Emergency Department Intervention "MOCAREDI": An outcome evaluation. Australas Emerg Nurs J 2011;14:S12

39 Gandara BK, Leresche L, Mancl L. Patterns of salivary estradiol and progesterone across the menstrual cycle. Ann N Y Acad Sci 2007;1098:446-50.

40 Baker JM, Al-Nakkash L, Herbst-Kralovetz MM. Estrogen-gut microbiome axis: physiological and clinical implications. Maturitas 2017;103:45-53

41 Caporaso JG, Lauber CL, Walters WA, et al. Global patterns of 16S rRNA diversity at a depth of millions of sequences per sample. Proc Natl Acad Sci U S A 2011;108(Suppl 1):4516-22.

42 Sheedy JR, Ebeling PR, Gooley PR, et al. A sample preparation protocol for $1 \mathrm{H}$ nuclear magnetic resonance studies of water-soluble metabolites in blood and urine. Anal Biochem 2010;398:263-5.

43 Gratton J, Phetcharaburanin J, Mullish BH, et al. Optimized sample handling strategy for metabolic profiling of human feces. Anal Chem 2016;88:4661-8.
44 Beckonert O, Keun HC, Ebbels TMD, et al. Metabolic profiling, metabolomic and metabonomic procedures for NMR spectroscopy of urine, plasma, serum and tissue extracts. Nat Protoc 2007;2:2692-703.

45 Byrne ML, Horne S, O'Brien-Simpson NM, et al. Associations between observed parenting behavior and adolescent inflammation two and a half years later in a community sample. Health Psychol 2017;36:641-51

46 Bolyen E, Rideout JR, Dillon MR, et al. Reproducible, interactive, scalable and extensible microbiome data science using QIIME 2. Nat Biotechnol 2019;37:852-7.

47 Callahan BJ, McMurdie PJ, Rosen MJ, et al. DADA2: high-resolution sample inference from illumina amplicon data. Nat Methods 2016;13:581-3

48 Katoh K, Misawa K, Kuma K-ichi, et al. MAFFT: a novel method for rapid multiple sequence alignment based on fast Fourier transform. Nucleic Acids Res 2002;30:3059-66.

49 Price MN, Dehal PS, Arkin AP. FastTree 2--approximately maximumlikelihood trees for large alignments. PLoS One 2010;5:e9490.

50 Lin H, Peddada SD. Analysis of compositions of microbiomes with bias correction. Nat Commun 2020;11:3514

51 Mallick H, Mclver LJ, Rahnavard A. Multivariable association in population-scale Meta-omics studies, 2020. Available: http:// huttenhower.sph.harvard.edu/maaslin2

52 Oksanen J, Blanchet FG, Kindt R. Vegan: community ecology package, 2012. Available: https://cran.r-project.org/web/packages/ vegan/index.htm

53 McMurdie PJ, Holmes S. phyloseq: an R package for reproducible interactive analysis and graphics of microbiome census data. PLoS One 2013;8:e61217.

54 Wickham H. ggplot2: elegant graphics for data analysis. New York: Springer-Verlag, 2016. https://ggplot2.tidyverse.org

55 Rohart F, Gautier B, Singh A. mixOmics: an R package for 'omics feature selection and multiple data integration. bioRxiv 2017;108597.

56 Gratz KL, Roemer L. Multidimensional assessment of emotion regulation and dysregulation: development, factor structure, and initial validation of the difficulties in emotion regulation scale. $J$ Psychopathol Behav Assess 2004;26:41-54.

57 Beck A, Steer R, Brown G. Manual for the Beck depression Inventory-II. San Antonio, TX: Psychological Corporation, 1996.

58 Beck A, Streer R. Beck anxiety inventory manual. San Antonio, TX: Psychological Corporation, 1993.

59 Achenbach T, Rescorla L. Manual for the ASEBA adult forms \& profiles. Burlington: University of Vermont, Research Center for Children, Youth, and Families, 2003.

60 Giles G, Ireland P. Dietary questionnaire for epidemiological studies (version 3.2). Melbourne: Cancer Council Victoria, 1996.

61 The WHOQOL Group. The world Health organization quality of life assessment (WHOQOL): development and general psychometric properties. Soc Sci Med 1998;46:1569-85.

62 World Health Organization. Oral health surveys: basic methods. 5th edn. World Health Organization, 1987.

63 Schat ACH, Kelloway EK, Desmarais S. The physical health questionnaire (PHQ): construct validation of a self-report scale of somatic symptoms. J Occup Health Psychol 2005;10:363-81.

64 Greenwald R, Rubin A. Assessment of posttraumatic symptoms in children: development and preliminary validation of parent and child scales. Res Soc Work Pract 1999:9:61-75.

65 Berstein D, Fink L. Childhood trauma questionnaire: a retrospective self-report manual. San Antonio, TX: Psychological Corporation, 1998. 